\title{
GROUPS OF COMPLEXES OF A REPRESENTABLE LATTICE-ORDERED GROUP
}

\author{
by R. D. BYRD, J. T. LLOYD and J. W. STEPP
}

(Received 22 January, 1977)

1. Introduction. In $1954 \mathrm{~N}$. Kimura proved that each idempotent in a semigroup is contained in a unique maximal subgroup of the semigroup and that distinct maximal subgroups are disjoint [13] (or see [6, pp. 21-23]). This generalized earlier results of Schwarz [14] and Wallace [15]. These maximal subgroups are important in the study of semigroups. If $G$ is a group, then the collection $S(G)$ of nonempty complexes of $G$ is a semigroup and it is natural to inquire what properties of $G$ are inherited by the maximal subgroups of $S(G)$. There seems to be very little literature devoted to this subject. In [5, Theorem 2], with certain hypotheses placed on an idempotent, it was shown that if $G$ is a lattice-ordered group ("l-group") then a maximal subgroup of $S(G)$ containing an idempotent satisfying these conditions admits a natural lattice-order. The main result of this note (Theorem 1 ) is that if $G$ is a representable $l$-group and $E$ is a normal idempotent of $S(G)$ and a dual ideal of the lattice $G$, then the maximal subgroup of $S(G)$ containing $E$ admits a representable lattice-order.

2. Notation and terminology. The collection $S(G)$ of all nonempty complexes (subsets) of a group $G$ is a semigroup with respect to the binary operation $A B=$ $\{a b \mid a \in A$ and $b \in B\}$ for $A, B \in S(G)$. If $E$ is an idempotent in $S(G)$, then $H(E)$ denotes the maximal subgroup of $S(G)$ that contains $E$. A normal idempotent $E$ of $S(G)$ is an idempotent of $S(G)$ and normal subset of $G$. If $E$ is a normal idempotent of $S(G)$, then $T(E)=\{a E \mid a \in G\}$ is a subgroup of $H(E)$ and is isomorphic to $G$ modulo the kernel of the mapping $\gamma$ which sends $x$ to $x E$ for all $x \in G$. Moreover, if $A \in H(E)$, then $x A x^{-1} \in H(E)$ for all $x \in G$. If $1 \in E$, where $E$ is a normal idempotent of $S(G)$, then $T(E)=H(E)[4$, Proposition 4], and hence any property of $G$ that is preserved by homomorphic images will be inherited by $H(E)$. Consequently, the "more interesting" cases of maximal subgroups of $S(G)$ occur when the identity element of $G$ does not belong to the idempotent.

For the remainder of this note, we assume that $G$ is an $l$-group, $E$ is a normal idempotent of $S(G)$, and $\gamma$ is the mapping of $G$ into $H(E)$ given by $\gamma(x)=x E$ for all $x \in G$. For the standard definitions and results concerning $l$-groups the reader is referred to [1], [10], and [12]. An $l$-group $G$ is said to be representable if there exists an $l$-isomorphism of $G$ into a cardinal sum of totally ordered groups ("o-groups"). A dual ideal of $G$ is a nonempty subset $I$ of $G$ such that $a, b \in I, x \in G$, and $x \geq a$ imply $a \wedge b, x \in I$. A prime subgroup of $G$ is a convex $l$-subgroup such that if $a, b \in G^{+} \backslash M$, then $a \wedge b \in G^{+} \backslash M$.

Let $E$ be a dual ideal of $G$. For an element $A$ in $H(E)$, define $A \geq E$ if and only if $A \subseteq E$. Then $H(E)$ is an $l$-group with positive cone $\{A \mid A \geqslant E\}, T(E)$ is an $l$-subgroup of $H(E)$ with $a E \vee b E=(a \vee b) E$ and dually for all $a, b \in G$, and the kernel of $\gamma$ is an $l$-ideal of $G$ [5, Theorem 2]. It was shown in the proof of this theorem that if $A \in H(E)$, then $A \vee E=\cup(a \vee 1) E(a \in A)$. 
If $X$ and $Y$ are sets, then $X \backslash Y$ denotes the set of elements in $X$ but not in $Y$, and if $G$ is an $l$-group, then $G^{+}=\{g \in G \mid g \geq 1\}$.

3. Representability of $\mathbf{H ( E )}$. If $M$ is an $l$-subgroup of $G$ such that for every $g \in G$ with $g>1$ there exists an $a \in M$ with $g \geq a>1$, then $M$ is said to be dense in $G$. If $M$ is dense in $G$, then joins and meets in $M$ agree with those in $G$ [2, Lemma 10]. The proofs of the next two lemmas are straightforward and will be omitted.

LEMMA 1. Let $G$ and $M$ be l-groups, $\eta$ an l-homomorphism of $G$ onto $M$, and $E$ a dual ideal of $G$. Then $E^{*}=\eta(E)$ is a normal idempotent of $S(M)$, a dual ideal of $M$, and if $A \in H(E)$, then $\eta(A) \in H\left(E^{*}\right)$. Let $\eta^{*}: H(E) \rightarrow H\left(E^{*}\right)$ be given by $\eta^{*}(A)=\eta(A)$ for all $A \in H(E)$, and let $\gamma^{*}: M \rightarrow H\left(E^{*}\right)$ be the l-homomorphism given by $\gamma^{*}(x)=x E^{*}$ for all $x \in M$. Then $\eta^{*}$ is an l-homomorphism making the following diagram commute

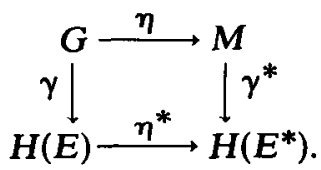

LemMA 2. If $E$ is a dual ideal of an o-group, then $H(E)$ is an o-group.

Lemma 3. If $E$ is a dual ideal of $G$, then $T(E)$ is dense in $H(E)$. Moreover, if $A \in H(E)$, then there is a $g \in G$ with $A \leq g E$.

Proof. Let $A \in H(E)$ such that $E<A$ and let $B$ be the inverse of $A$ in $H(E)$. We may assume $A \notin T(E)$. Then we have $B<E<A$ and $B \notin T(E)$. Thus $B<b E$ for all $b \in B$. Now

$$
B=B \wedge E<b E \wedge E=(b \wedge 1) E \leq E
$$

for all $b \in B$. Suppose that $(b \wedge 1) E=E$ for all $b \in B$. Then $E=(b \wedge 1) E \leq b E$ for all $b \in B$ and so $B=B E=\bigcup_{b \in B} b E \subseteq E$, a contradiction. Thus $B<(b \wedge 1) E<E$ for some $b \in B$ and so $E<(b \wedge 1)^{-1} E<A$. Therefore $T(E)$ is dense in $H(E)$. The second statement of the lemma is clear.

An element $b$ of $G$ is called basic if $b>1$ and $\{x \mid x \in G$ and $1<x \leq b\}$ is totally ordered. A subset $B$ of $G$ is a basis if $B$ is a maximal set of (pairwise) disjoint elements in $G$ and each $b$ in $B$ is basic. An $l$-group $G$ has a basis if and only if for every $g \in G$ with $g>1, g$ exceeds a basic element [7, Theorem 5.1]. An atom is an element of $G$ that covers 1 .

COROLlaRY 1. If $E$ is a dual ideal of $G, \gamma$ is one-to-one, and $b$ is basic (respectively, an atom), then $b E$ is basic (respectively, an atom) in $H(E)$. Hence, if $G$ has a basis, then $H(E)$ has a basis.

THEOREM 1. If $E$ is a dual ideal of $G$ and $G$ is representable, then $H(E)$ is representable. 
Proof. By [8, Lemma 3] it suffices to show that for each strictly positive element $A$ of $H(E)$, there is an $l$-homomorphism $\eta^{*}$ from $H(E)$ into an $o$-group such that $\eta^{*}(A) \neq 1$. Since $T(E)$ is dense in $H(E)$, it suffices to take $A=a E$ where $a>1$. We first consider the case in which the mapping $\gamma$ is one-to-one. We assert that in this case $E \subseteq G^{+}$. If $b \in E$, then $b E \subseteq E$ and so $b E \geq E$. Thus $b E=b E \vee E=(b \vee 1) E$. Since $\gamma$ is one-to-one, $b=b \vee 1 \epsilon$ $G^{+}$. Now suppose that $a \leq e$ for every $e \in E$. Then, if $e \in E, e=e_{1} e_{2}$, where $e_{1}, e_{2} \in E$ and $a \leq e_{1}$. Hence $a e_{2} \leq e_{1} e_{2}=e$ and so $e \in a E$. Since $a E$ is positive, $a E \subseteq E$. But then $a E=E$ contrary to the choice of $a E$. It follows that $a \wedge e<a$ for some $e \in E$. Since $G$ is representable, there is a minimal prime subgroup $M$ of $G$ which is normal [3, Theorem 3.1], $G / M$ is an o-group, and $(a \wedge e) M<a M$. Consider the following commutative diagram

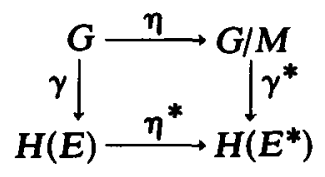

where $\eta$ is the canonical mapping, $E^{*}=\eta(E), \gamma^{*}(x M)=x M E^{*}$ for every $x M \in G / M$, and $\eta^{*}(A)=\eta(A)$ for every $A \in H(E)$. By Lemma $1, \eta^{*}$ is an $l$-homomorphism and by Lemma $2, H\left(E^{*}\right)$ is an o-group. Now $\eta(a) \wedge \eta(e)=\eta(a \wedge e)<\eta(a)$ and since $G / M$ is totally ordered, we have that $\eta(e)<\eta(a)$. If $f \in E$, then $\eta(a f)=\eta(a) \eta(f) \geq \eta(a)>\eta(e)$. Therefore $\eta(a E) \neq \eta(E)$ and so $\eta^{*}(a E) \neq E^{*}$. Thus $H(E)$ is representable.

We next consider the general case. Let $K$ denote the kernel of $\gamma$ and consider the following commutative diagram

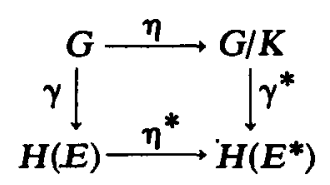

where $\eta, E^{*}, \gamma^{*}$, and $\eta^{*}$ are as given above. We assert that $\gamma^{*}$ is one-to-one. If $x, y \in G$ with $\gamma^{*} \eta(x)=\gamma^{*} \eta(y)$, then $\eta(x) E^{*}=\eta(y) E^{*}$ and so $x K E=y K E$. Since $K E=E$, we have that $x E=y E$ and so $\eta(x)=\eta(y)$. Now $G / K$ is representable and therefore, by the previous case, there is an $l$-homomorphism $\theta$ of $H\left(E^{*}\right)$ into an o-group such that $\theta \eta^{*}(a E) \neq 1$. It now follows that $H(E)$ is representable.

An l-group $G$ is said to be epi-Archimedean (or hyper-Archimedean) if each $l$-homomorphic image of $G$ is Archimedean. In [11, Theorem 1.1] five conditions are given each of which is equivalent to the epi-Archimedean property.

THEOREM 2. If $E$ is a dual ideal of $G$ and $G$ is epi-Archimedean, then $H(E)$ is Archimedean.

Proof. Let $A, B \in H(E)$ such that $A>E$ and $B>E$. By Lemma 3, there exist $a, b \in G^{+}$ such that $A \geq a E>E$ and $b E \geq B$. Since $T(E)$ is an $l$-homomorphic image of $G, T(E)$ is Archimedean. Thus there exists a positive integer $n$ such that $a^{n} E \$ b B$. Hence $A^{n} \ddagger B$ and so $H(E)$ is Archimedean. 
4. Examples. In this section we give some examples to illustrate the scope and limitations of our results.

Example 1. Let $G=\sum_{i=1}^{n} \mathbf{Q}$ be the cardinal product of $n$ copies of the rational numbers $\mathbf{Q}$ and let $E=\left\{\left(a_{1}, a_{2}, \ldots, a_{n}\right) \mid a_{i}>0, i=1,2, \ldots, n\right\}$. Then $E$ is a normal idempotent and a dual ideal of $G$. In this case $H(E)$ is $l$-isomorphic to $\sum_{i=1}^{n} \mathbf{R}$, the cardinal sum of $n$ copies of the real numbers.

In the above example $E$ is the collection of units. In any $l$-group the collection of units is a normal subset, and, when nonempty, is a dual ideal. However, in general, the collection of units will not be an idempotent.

EXAMPLE 2. Let $M$ be a normal proper prime subgroup of $G$ such that $G / M$ has no atoms. Then $E=U(x M)(x M>M)$ is a normal idempotent and a dual ideal. Moreover, $M$ is the kernel of $\gamma$. If $G$ is Archimedean and $M$ is not a maximal ideal of $G$, then $H(E)$ is not Archimedean.

If $G$ is an $l$-subgroup of an $l$-group $M$, then $M$ is said to be an a-extension of $G$ provided that for every $1<a \in M$ there exists $g \in G$ and there exist positive integers $m$ and $n$ such that $g \leq a^{m}$ and $a \leq g^{n}$. If $G$ is an $l$-subgroup of $M$, then $M$ is an $a$-extension of $G$ if and only if the mapping $C \rightarrow C \cap G$ is a one-to-one mapping of the lattice of convex $l$-subgroups of $M$ onto the lattice of convex $l$-subgroups of $G$ [9, Theorem 2.1]. Our next example shows that even when $\gamma$ is one-to-one, $H(E)$ need not be an a-extension of $G$. The example also shows that when $\gamma$ is one-to-one and $G$ is epi-Archimedean, then $H(E)$ need not be epi-Archimedean, and thus Theorem 2 is in some sense the best possible.

Example 3. Let $G=\left\{f: \mathbf{Z}^{+} \rightarrow \mathbf{R} \mid\right.$ there exists $N \in \mathbf{Z}^{+}$with $f(n)=f(N)$ for all $\left.n \geq N\right\}$, with $f \leq g$ if $f(n) \leq g(n)$ for all $n \in \mathbf{Z}^{+}$, and function addition is the group operation. Let $E=\left\{f \in G \mid f(n)>0\right.$ for all $\left.n \in \mathbf{Z}^{+}\right\}$. Then $E$ is a normal idempotent and a dual ideal. In fact, $E$ is the collection of units of $G$. Let $A=\left\{f \in G \mid f(n)>1 /(n+1)\right.$ for all $\left.n \in \mathbf{Z}^{+}\right\}$. Then $A \in H(E)$ and $E<A$. If there exists $g \in G$ and a positive integer $m$ with $g E \leq A^{m}$ then there is a positive integer $N$ with $g(k) \leq 0$ if $k \geq N$. But if $g(k) \leq 0$ for $k \geq N$, then $A \neq g^{n} E$ for all $n \in \mathbf{Z}^{+}$. Thus $H(E)$ is not an $a$-extension of $G$.

\section{REFERENCES}

1. G. Birkhoff, Lattice Theory (Amer. Math. Soc., 1967).

3. R. D. Byrd, Complete distributivity in lattice-ordered groups, Pacific J. Math. 20 (1967), 423-432.

4. R. D. Byrd, J. T. Lloyd and J. W. Stepp, Groups of complexes of a group, J. Natur. Sci. and Math. 15 (1975), 83-87.

5. R. D. Byrd, J. T. Lloyd and J. W. Stepp, Groups of complexes of a lattice-ordered group, Sym. Math. 21 (1977) 525-528.

6. A. H. Clifford and G. B. Preston, The algebraic theory of semigroups Vol. I, Amer. Math. Soc. Mathematical Surveys 7 (Providence, R.I., 1961). 
7. P. F. Conrad, Some structure theorems for lattice-ordered groups, Trans. Amer. Math. Soc. 99 (1961), 212-240.

8. P. F. Conrad, The relationship between the radical of a lattice-ordered group and complete distributivity, Pacific J. Math. 14 (1964), 493-499.

9. P. F. Conrad, Archimedean extensions of lattice-ordered groups, J. Indian Math. Soc. 30 (1966), 131-160.

10. P. F. Conrad, Lattice-ordered groups, Lecture Notes (Tulane University, 1970).

11. P. F. Conrad, Epi-Archimedean groups, Czechoslovak Math. J. 24 (1974), 192-218.

12. L. Fuchs, Partially ordered algebraic systems (Pergamon Press, 1963).

13. N. Kimura, Maximal subgroups of a semigroup, Kōdai Math. Sem. Rep. 1954 (1954), 85-88.

14. S. Schwarz, Zur Theorie der Halbgruppen (Slovakian, German summary), Sbornik prác Prirodovedekej Fakulty Slovenskej University v Bratislave No 6 (1943).

15. A. D. Wallace, A note on mobs II, An. Acad. Brasil Ci. 25 (1953), 335-336.

UNIVERSITY OF HOUSTON 